Ambiente \& Água - An Interdisciplinary Journal of Applied Science
ISSN 1980-993X - doi:10.4136/1980-993X
www.ambi-agua.net
E-mail: ambi-agua@agro.unitau.br

\title{
Estimativa de componentes do balanço de radiação em diferentes tipos de uso e cobertura do solo
}

\author{
doi: 10.4136/ambi-agua.1273
}

Received: 07 Jan. 2014; Accepted: 30 Apr. 2014

\author{
Fernando da Silva Sallo ${ }^{1}$; Mauro Sergio de França ${ }^{2}$; Denes Martins de Morais ${ }^{1}$; \\ Rodicrisller Rodrigues ${ }^{1}$; Marcelo Sacardi Biudes ${ }^{1}$ \\ ${ }^{1}$ Universidade Federal de Mato Grosso, Cuiabá, MT, Brasil \\ Programa de Pós-Graduação em Física Ambiental \\ ${ }^{2}$ Instituto Federal de Educação, Ciência e Tecnologia de Mato Grosso, Sorriso, MT, Brasil \\ *Autor correspondente: e-mail: fdss88@ gmail.com, \\ mauro.franca@srs.ifmt.edu.br, senedbr@yahoo.com.br, \\ rodi.067@gmail.com, marcelo.biudes@gmail.com
}

\section{RESUMO}

Estudos sobre o balanço de radiação em áreas de expansão agropecuária, especialmente em estados agroexportadores como Mato Grosso, são relevantes para a compreensão da interação atmosfera-biosfera. Este estudo estimou alguns componentes do balanço de radiação em áreas distintas (floresta secundária, reflorestamento com eucaliptos e cultivos agrícolas) no município de Lucas do Rio Verde/MT com base em magens do satélite Landsat 5 - TM. As imagens escolhidas foram de 20/01 e 26/04 do ano de 2010. As imagens foram processadas utilizando o algoritmo SEBAL para o cálculo dos valores do saldo de radiação instantâneo $\left(\mathrm{Rn}_{\text {inst }}\right)$, albedo e NDVI. Os resultados evidenciaram alta variabilidade no $\mathrm{Rn}_{\text {inst }}$, albedo e NDVI tanto entre as distintas coberturas quanto para as diferentes datas. As áreas de floresta secundária e reflorestamento de eucaliptos apresentaram magnitudes similares, enquanto as áreas com cultivos agrícolas os valores apresentam relações intrínsecas referentes aos estádios de desenvolvimento com complexa interação radiativa.

Palavras-chave: interação biosfera-atmosfera, sensoriamento remoto, uso da terra.

\section{Estimation of components of the radiation balance of different types of land use and land cover}

\begin{abstract}
Studies on the radiation balance in areas of agricultural expansion, especially in agro-exporting states such as Mato Grosso, are relevant to understanding the atmosphere-biosphere interaction. This study estimated some components of the radiation balance in different areas (secondary forest, reforestation with eucalyptus trees and agricultural crops) in the city of Lucas do Rio Verde/MT using Landsat 5 - TM images. The images chosen were acquired on January 20 and April 26 of 2010. The images were processed using the SEBAL algorithm to calculate the values of the instantaneous radiation balance $\left(R n_{\text {inst }}\right)$, albedo and NDVI. The results showed high variability of $\mathrm{Rn}_{\text {inst }}$, albedo and NDVI considering both different coverages and dates. The areas of secondary forest and
\end{abstract}


reforestation of eucalyptus showed similar magnitudes, while areas with agricultural crops had values with intrinsic relations for the stages of development with complex radiative interaction.

Keywords: biosphere-atmosphere interaction, remote sensing, land use.

\section{INTRODUÇÃO}

A agricultura compreende uma das principais atividades econômicas da região médio-norte do estado de Mato Grosso, com destaque para a produção de soja e milho, sendo Nova Mutum, Lucas do Rio Verde, Sorriso e Sinop os principais municípios produtores. Esta região é caracterizada por dois biomas, o cerrado e o cerradão (ou zona de transição Cerrado-Amazônia), com solos e clima (com destaque para a intensidade e regularidade pluviométrica) que favorecem uma safra a mais que outras regiões do país conhecido como produção do "milho safrinha" (Beckmann e Oliveira, 2010).

A expansão da produtividade agrícola tem contribuído para modificar a paisagem natural, suprimindo enormes áreas de cerrado e florestas, resultando em extensas áreas desmatadas, que proporcionaram diversas mudanças ambientais, entre elas, alterações no balanço de radiação e de energia da superfície interferindo na temperatura e na umidade do ar e nos processos de trocas gasosas entre a superfície e atmosfera devido à contínua mudança na arquitetura da vegetação, e consequentemente na condutância da superfície ao transporte de vapor d'água, no sistema radicular e no albedo da superfície (Dubreuil e Delahaye, 2010; Souza et al., 2013).

Essa modificação do uso do solo pode ser mensurada e/ou analisada através do sensoriamento remoto, que possibilita a variabilidade espacial em larga escala, o monitoramento espacial e temporal dos diversos biomas que constituem a superfície e a identificação, em tempo real, de mudanças que venham a ocorrer (Gomes et al., 2009), sendo essa técnica uma opção a ser explorada, dado o seu caráter multiespectral, que possibilita análise de componentes agrometeorológicos, como o albedo, índices de vegetação e evapotranspiração (Seixas et al., 2010).

Um dos algoritmos que se destacam no sensoriamento remoto, usado em diversos países é o SEBAL (Surface Energy Balance Algorith for Land), desenvolvido por Bastiaanssen (1995), que é muito utilizado em estudos dos fluxos de calor latente e sensível à superfície e vem sendo aplicado por diferentes pesquisadores em várias partes do mundo (Bastiaanssen et al., 1998; Bastiaanssen, 2000; Morse et al., 2000; Allen et al., 2002). O mesmo é alimentado com imagens de satélite e poucos dados de superfície, para estimar os fluxos de calor latente, sensível e no solo. O uso do SEBAL tem a grande vantagem de proporcionar o balanço de energia à superfície de maneira efetiva e econômica. Possibilita uma grande cobertura espacial e, dependendo do sensor orbital que o alimenta com dados dos canais reflectivos e termais, pode apresentar grande resolução espacial.

Desse modo, o presente estudo buscou estimar o saldo de radiação instantâneo $\left(\mathrm{Rn}_{\text {inst }}\right)$, albedo e NDVI em três áreas distintas, floresta secundária, reflorestamento com eucaliptos e cultivos agrícolas no município de Lucas do Rio Verde/MT em 2010, sendo que essas variáveis ambientais são vitais para o entendimento das trocas entre a superfície e atmosfera.

\section{MATERIAL E MÉTODOS}

\section{1. Área de estudo}

O município de Lucas do Rio Verde/MT tem uma população de 45.556 habitantes e uma extensão territorial de $3.664 \mathrm{~km}^{2}$ (IBGE, 2010), localizado no médio norte mato-grossense (figura 1). O clima predominante é o Aw para Köppen, com temperatura média de $25^{\circ} \mathrm{C}$ e precipitação anual de $2.300 \mathrm{~mm}$. A vegetação é constituída por cerrado, arbóreo denso 
(cerradão) e matas ciliares, com predomínio de relevo plano com solos argilosos e areno-argilosos, com predominância de latossolos vermelho amarelo distrófico (Ferreira, 2001).

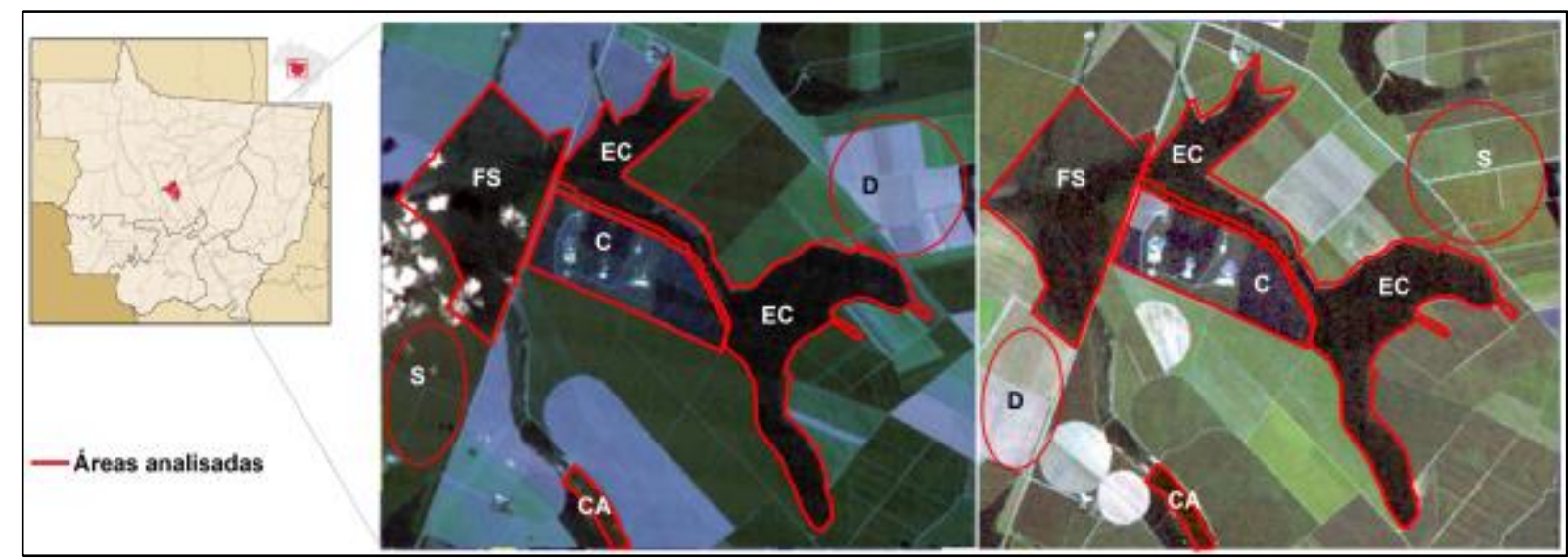

Figura 1. Área de estudo: Município de Lucas do Rio Verde/MT. Dia 20/01/2010: cultura + construções (C); corpo d'água (CA); solo semiexposto (D); eucalipto (EC); floresta secundária (FS); soja (S). Dia 26/04/2010: cultura + construções (C); corpo d'água (CA); milho (S); eucalipto (EC); floresta secundária (FS); solo semiexposto (D). Os dados de uso/cobertura do solo foram coletados in situ.

Fonte: Adaptado de http://wikimedia.org/MatoGrosso_Municip_LucasdoRioVerde.svg/200px-.svg.png>. Acesso em 30 dez. 2013.

A área selecionada para o estudo teve como referência central as coordenadas

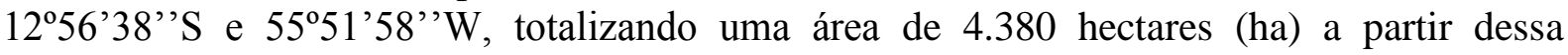
referência, com altitude média de $390 \mathrm{~m}$, a cerca de $10 \mathrm{~km}$ da sede local. A tabela 1 mostra a composição predominante da área.

Tabela 1. Distribuição das classes de uso do solo na área selecionada (ha).

\begin{tabular}{lc}
\hline \multicolumn{1}{c}{ Classes de uso do solo } & Hectares (ha) \\
\hline Floresta secundária de transição (FS) & 656 \\
Reflorestamento com eucaliptos (EC) & 990 \\
Lavoura de milho e/ou soja (S) & 2.199 \\
$\begin{array}{l}\text { Armazéns e/ou construções (C), corpos } \\
\text { d'águas (CA) e solo exposto (D) }\end{array}$ & 535 \\
\hline \multicolumn{1}{c}{ Total } & $\mathbf{4 . 3 8 0}$ \\
\hline
\end{tabular}

\subsection{Pré-processamento digital de imagens multiespectrais}

As duas imagens da área de estudo foram escolhidas levando em consideração a disponibilidade de dias sem ou pouca cobertura de nuvens e a época da lavoura da soja (20/01/2010) e do milho (26/04/2010) na região.

O pré-processamento envolveu as seguintes etapas: recorte das imagens obtidas do Landsat 5 - TM da área estudo; empilhamento das diversas camadas da imagem em um único arquivo de imagem; ortorretificação e georreferenciamento, que nesse caso adotou-se o World Geodetic System 84 (WGS84) como padrão. O processamento da imagem envolveu as seguintes etapas: conversão dos dados da imagem de números digitais para radiância 
espectral; obtenção da refletância, do índice de vegetação Soil-adjusted Vegetation Index (SAVI) e do índice de área foliar (IAF); estimativa do albedo planetário e do albedo corrigido (ou da superfície); obtenção do The Normalized Difference Vegetation Index (NDVI), estimativa da radiação de onda longa emitida e da temperatura da superfície; e por fim, da estimativa do saldo de radiação.

\subsection{Cálculo do Rn}

$\mathrm{O} \mathrm{Rn}_{\text {inst }}\left(\mathrm{W} \mathrm{m}^{-2}\right)$ foi calculado pelo o algoritmo SEBAL (Bastiaanssen et al., 1998) à superfície foi obtido por meio da Equação 1:

$$
R n=(1-\propto) R_{S \downarrow}+R_{L \downarrow}-R_{L \uparrow}-\left(1-\varepsilon_{0}\right) R_{L \downarrow}
$$

em que:

Rs $\downarrow$ é a radiação de onda curta incidente em $\mathrm{W} \mathrm{m}^{-2}$,

$\alpha$ é albedo da superfície, adimensional,

$\mathrm{R}_{\mathrm{L} \downarrow}$ é a radiação de onda longa incidente da atmosfera $\mathrm{W} \mathrm{m}^{-2}$,

$\mathrm{R}_{\mathrm{L} \uparrow}$ é a radiação de onda longa emitida por cada pixel em $\mathrm{W} \mathrm{m} \mathrm{m}^{-2} \mathrm{e}$

$\varepsilon_{0}$ é a emissividade do de cada pixel.

Para transformação do número digital (ND) de cada pixel em radiância espectral $\left(\mathrm{L}_{\lambda \mathrm{i}}\right)$ que representa a energia solar refletida por cada pixel por unidade de área, tempo, ângulo sólido e de comprimento de onda, medida em nível do satélite, sendo obtido pela Equação 2 (Chander et al., 2003):

$$
L_{\lambda i}=a_{i}+\frac{b_{i}-a_{i}}{255} N D
$$

em que:

a e b são as radiâncias mínimas e máximas obtidas mediante processo de calibração do Landsat 5 - TM;

$\mathrm{L}_{\lambda \mathrm{i}}$ radiância espectral de cada banda;

ND número digital (número inteiro entre 0 a 255) e

i correspondem às bandas $(1,2,3, \ldots$ e 7$)$ do Landsat 5 - TM.

A fase seguinte foi o cálculo da refletância monocromática no topo da atmosfera $\left(\rho_{\lambda_{\mathrm{i}}}\right)$. tal variável é estimada pela razão entre o fluxo de radiação solar refletido e o fluxo de radiação solar incidente utilizou-se a Equação 3 (Chander e Markham, 2003):

$$
\mathrm{P} \lambda \mathrm{i}=\frac{\pi \cdot L_{\lambda i}}{K_{\lambda i} \cdot \cos Z \cdot d r}
$$

em que:

$\mathrm{L}_{\lambda_{\mathrm{i}}}$ é a radiância monocromática em $\mathrm{W} \mathrm{m}^{-2} \mathrm{sr} \mu \mathrm{m}$;

$\mathrm{K}_{\lambda \mathrm{i}}$ é a irradiância solar monocromática em $\mathrm{W} \mathrm{m}^{-2} \mu \mathrm{m}$;

Z é o ângulo zenital solar, e

dr é o inverso do quadrado da distância relativa Terra-Sol.

A partir dessas duas cartas anteriores é possível estimar o albedo planetário de cada pixel, ainda sem correção atmosférica, conforme a Equação 4:

$$
\alpha_{\text {plan }}=w_{\lambda 1} \cdot \rho_{\lambda 1}+w \cdot \rho_{\lambda 2}+w \cdot \rho_{\lambda 3}+w_{\lambda 4} \cdot \rho_{\lambda 4}+w_{\lambda 5} \cdot \rho_{\lambda 5}+w_{\lambda 7} \cdot \rho_{\lambda 7}
$$


em que:

$\alpha_{\text {plan }}$ é o albedo planetário sem correção;

$\mathrm{W}_{\lambda}$ é o peso de cada banda dado pela razão entre $\mathrm{K}_{\lambda \mathrm{i}}$ de cada banda e o somatório de todos os valores de $\mathrm{K}_{\lambda \mathrm{i}}$.

Assim, o albedo da superfície $\left(\alpha_{\text {sup }}\right)$ que representa o albedo corrigido dos efeitos da atmosfera, é dado pela Equação 5 (Bastiaanssen et al., 1998):

$$
\alpha_{\text {sup }}=\left(\frac{\alpha_{\text {plan }}-a}{\tau_{s w^{2}}}\right)
$$

A reflectância da atmosfera adotado 0,03 (Silva et al., 2005a) $\tau_{\text {Sw }}$ é a transmissividade atmosférica proposto por Allen et al. (2007).

O Índice de Vegetação da Diferença Normalizada (NDVI) foi calculado pela Equação 6 de Huete et al. (2002).

$$
N D V I=\frac{\rho_{I V P}-\rho_{V E R}}{\rho_{I V P}+\rho_{V E R}}
$$

em que:

$\rho_{I V P}=$ reflectância do infravermelho próximo;

$\rho_{V E R}=$ reflectância do vermelho.

O Índice de Vegetação Ajustado aos efeitos do Solo (SAVI) foi utilizado para estimativa da emissividade e para avaliar impactos decorrentes da ocupação humana (Huete, 1988), conforme a Equação 7:

$$
S A V I=\frac{(1+L)\left(\rho_{4}-\rho_{3}\right)}{\left(L+\rho_{4}+\rho_{3}\right)}
$$

em que:

L é uma constante de ajuste cujo valor usado foi de 0,1 (Allen et al., 2007; Silva et al., 2011).

Outro índice de vegetação utilizado foi o IAF (Índice de Área Foliar), definido pela razão entre a área foliar de toda a vegetação por unidade de área utilizada por essa vegetação, constituindo um indicador da biomassa de cada pixel, que foi computado pela Equação 8 (Allen et al., 2007):

$$
I A F=-\frac{\ln \left(\frac{0,69-S A V I}{0,59}\right)}{0,91}
$$

Uma vez que os pixels não emitem radiação eletromagnética como um corpo negro, ao se calcular a temperatura da superfície, necessita-se calcular sua emissividade $\left(\varepsilon_{N B}\right)$. Para tanto, fez-se uso da expressão parametrizada pela Equação 9 (Allen et al., 2007):

$$
\varepsilon_{N B}=0,97+0,0033 . I A F
$$

Para calcular a radiação de onda longa emitida por cada pixel deve-se considerar a emissividade $\left(\varepsilon_{0}\right)$ em todo o domínio da onda longa (4 a $\left.100 \mu \mathrm{m}\right)$, representada pela Equação 10:

$$
\varepsilon_{0}=0,95+0,01 \cdot I A F
$$

Essas duas últimas equações são válidas para SAVI $>0$ e IAF $\leq 3$. Para pixels com $\mathrm{IAF} \geq 3, \varepsilon_{\mathrm{NB}}=\varepsilon_{0}=0,98$ e para corpos d'água SAVI $<0$ consideram-se $\varepsilon_{\mathrm{NB}}=0,99$ e $\varepsilon_{0}=0,985$ (Allen et al., 2007). 
A temperatura na superfície (Ts) é um das mais importantes variáveis para o cálculo do $\mathrm{Rn}_{\text {inst }}$, por meio da Lei de Planck invertida, segundo Markham e Barker (1986), Ts é pela Equação 11:

$$
T_{S}=\frac{K_{2}}{\ln \left(\frac{\varepsilon_{N B} K_{1}}{L_{\lambda, 6}}+1\right)}
$$

em que:

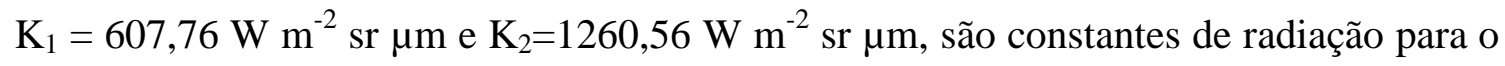
Landsat 5.

A partir de Ts é possível estimar a radiação de onda longa emitida $\left(\mathrm{R}_{\mathrm{L}, \mathrm{inc}}\right)$, usando a equação de Stefan-Boltzmann, pela Equação 12:

$$
R_{L \uparrow}=\varepsilon_{0} \sigma T_{S}^{4}
$$

Para a radiação de onda longa emitida pela atmosfera $\left(\mathrm{R}_{\mathrm{L}, \mathrm{emit}}\right)$ é estimada usando a equação acima, porém substituindo $\varepsilon_{0}$ por $\varepsilon_{a}$, o qual segundo Bastiaanssen et al. (1998) é dado pela Equação 13:

$$
\varepsilon_{a}=a\left(-\ln \tau_{s w}\right)^{b}
$$

em que:

a e b são coeficientes de calibração cujos valores, de acordo com Allen et al. (2007) são iguais, respectivamente, a 0,85 e 0,09 .

A radiação de onda curta incidente (Rs) que atinge a superfície terrestre em condição de céu claro, para imagens com área espacial menor que $25.000 \mathrm{~km}^{2}$, pode ser obtida pela Equação 14 (Allen et al., 2007):

$$
R_{S \downarrow}=S \cdot \cos Z \cdot d r \cdot \tau_{s w}
$$

em que:

$S$ é a constante solar,

$1367 \mathrm{~W} \mathrm{~m}^{-2}$,

$Z$ é o ângulo zenital solar,

$d r$ é o inverso do quadrado da distância relativa Terra-Sol,

$\tau_{S W}$ transmissividade atmosférica.

\section{RESULTADOS E DISCUSSÃO}

As variáveis $R n_{\text {inst }}$, albedo e NDVI estão inter-relacionados, uma vez que $\mathrm{Rn}_{\text {inst }}$ é diretamente dependente do albedo e o índice NDVI influencia na temperatura de superfície que por sua vez relaciona-se diretamente com $\mathrm{Rn}_{\text {inst }}$ (Tian et al., 2012). Os valores médios, mínimos e máximos obtidos do $\mathrm{Rn}_{\text {inst }}$, albedo e NDVI sobre todo o recorte são apresentados na Tabela 2.

As magnitudes dos valores médios, mínimos e máximos de $\mathrm{Rn}_{\text {inst }}$ diferem em razão do ângulo zenital solar, que é maior no mês de janeiro quando comparado ao mês de abril na área em análise, evidência encontrada por Gusmão et al. (2012) na Ilha do Bananal.

Tal diferença está associada também aos tipos de cobertura do solo e ao predomínio espacial dessa cobertura (Oliveira e Morais, 2013). Como nas imagens predominam floresta 
secundária (FS) e eucalipto (EC), os valores médios de $\mathrm{Rn}_{\text {inst, }}$ albedo e NDVI tendem aos valores encontrados para estas áreas.

Tabela 2. Valor médio, mínimo e máximo das variáveis para nos recortes em 20/01 e 26/04.

\begin{tabular}{lcccccc}
\hline Variáveis & 20/Jan. & 20/Jan. & 20/Jan. & 26/Abr. & 26/Abr. & 26/Abr. \\
\hline & Médio & Mínimo & Máximo & Médio & Mínimo & Máximo \\
$\begin{array}{l}\mathbf{R n}_{\text {inst }} \\
\left(\mathrm{W} \mathrm{m}^{-2}\right)\end{array}$ & 591,4 & 492 & 710,6 & 526,2 & 435,3 & 625,6 \\
$\begin{array}{l}\text { Albedo } \\
(\%)\end{array}$ & 17 & 5 & 43 & 15 & 3 & 48 \\
$\mathbf{N D V I}$ & 0,75 & $-0,23$ & 0,83 & 0,64 & $-0,13$ & 0,82 \\
\hline
\end{tabular}

A Tabela 3 mostra os valores médios obtidos para as diversas nas áreas analisadas para o período selecionado.

Tabela 3. Valor médio das variáveis para as áreas nos dias 20/01 e 26/04, respectivamente.

\begin{tabular}{|c|c|c|c|c|c|c|}
\hline \multirow{2}{*}{ Variáveis } & \multicolumn{5}{|c|}{ Classes de uso do solo* } & \multirow[b]{2}{*}{ CA } \\
\hline & FS & EC & $\mathbf{C}$ & D & $\mathbf{S}$ & \\
\hline $\begin{array}{l}\mathbf{R n}_{\text {inst }} \\
\left(\mathrm{W} \mathrm{m}^{-2}\right)\end{array}$ & $644 / 566$ & $649 / 570$ & $\begin{array}{l}622 / 512(385 / 370 \mathrm{em} \\
\text { construções) }\end{array}$ & $516 / 494$ & $574 / 458$ & $708 / 620$ \\
\hline Albedo (\%) & $12 / 11,7$ & $11 / 10,3$ & $\begin{array}{l}\text { 12/11,5 (38/42 em } \\
\text { construçôes) }\end{array}$ & $20 / 22$ & $21 / 19,5$ & $0,05 / 0,04$ \\
\hline NDVI & $0,66 / 0,69$ & $0,70 / 0,72$ & $\begin{array}{l}0,67 / 0,675(0,17 / 0,13 \\
\text { em construções) }\end{array}$ & $0,18 / 0,82$ & $0,83 / 0,37$ & $-0,13 /-0,1$ \\
\hline
\end{tabular}

*Nota: FS (Floresta secundária de transição); EC (Reflorestamento com eucaliptos); C (Armazéns e/ou construções); D (Solo exposto); S (Soja/Milho); CA (Corpos d'águas).

O maior valor de albedo para as duas imagens foi verificado na área S (26/04), que estava cultivada com milho, por conseguinte resultou em menor $\mathrm{Rn}_{\text {inst }}$, sugerindo que neste estádio fenológico o milho necessita de pouca energia disponível $(\mathrm{Rn})$ para seu crescimento. Alguns estudos mostraram que o albedo medido em estádios fenológicos intermediário de milho é ligeiramente maior que o albedo medido para solo exposto (Jacobs e Van Pul, 1990; Silva Neto et al., 2008). Esses valores de albedo na área $\mathrm{S}$ possuem, em média, a mesma magnitude, $21 \%$ e $23 \%$, dos obtidos por Jacobs e Van Pul (1990) e Silva Neto et al. (2008), em lavoura do milho, de 0,20 e 0,18 respectivamente.

As áreas D e S (soja) no dia 20/01 apresentaram valores similares de albedo (Tabela 3), sugerindo que a cultura de soja neste estádio fenológico exige menor energia disponível (Rn) para seu desenvolvimento. A variação do albedo durante todo o desenvolvimento da cultura de soja está em média entre 14 e 26\% (Fontana et al., 1991; Souza et al., 2010). Estudos de Souza et al. (2010) registraram os maiores valores de albedo no estádio de frutificação da cultura de 24\%, no estado do Pará. Tomando como base o dia da imagem (20/01) e o local de estudo, é muito provável que a área estivesse com a soja em período de final de frutificação (conferindo com o calendário da região), evidenciando que os dados obtidos pelo SEBAL são bem reais aos fatos observados em campo. 
Os valores de NDVI para abril na área $\mathrm{S}$ foram maiores que janeiro (área D), visto que em abril a cultura de milho apresentava-se em pleno crescimento, com elevado conteúdo de biomassa se comparada a pré-colheita da soja em janeiro. Para o milho o índice NDVI pode variar de aproximadamente 0,1 (dias pós-semeadura) a 1,1 (próximo à colheita) (Kar e Kumar, 2010). Os valores obtidos, em abril, foram de 0,82 , que evidencia cultura em frutificação. Os valores mínimos de NVDI e albedo foram encontrados nas áreas CA. A magnitude destes valores podem sofrer variações, que dependem do ângulo de incidência dos raios solares, profundidade do corpo d'água e das propriedades físico-químicas da água tais como: sólidos suspensos, nível de eutrofização, profundidade do corpo d'água entre outros (Gusmão et al., 2012). Sendo o $\mathrm{Rn}_{\text {inst }}$ inversamente proporcional ao albedo em corpos d'água, os valores do $\mathrm{Rn}_{\text {inst }}$ são máximos nesses locais, respectivamente em 26/04 e 20/01 (Tabela 2). Os resultados obtidos nas imagens (Figura 2: a até f) mostraram que nas áreas FS e EC os componentes $\mathrm{Rn}_{\text {inst }}$, albedo e NDVI não se alteraram bruscamente, comparado com as outras áreas.
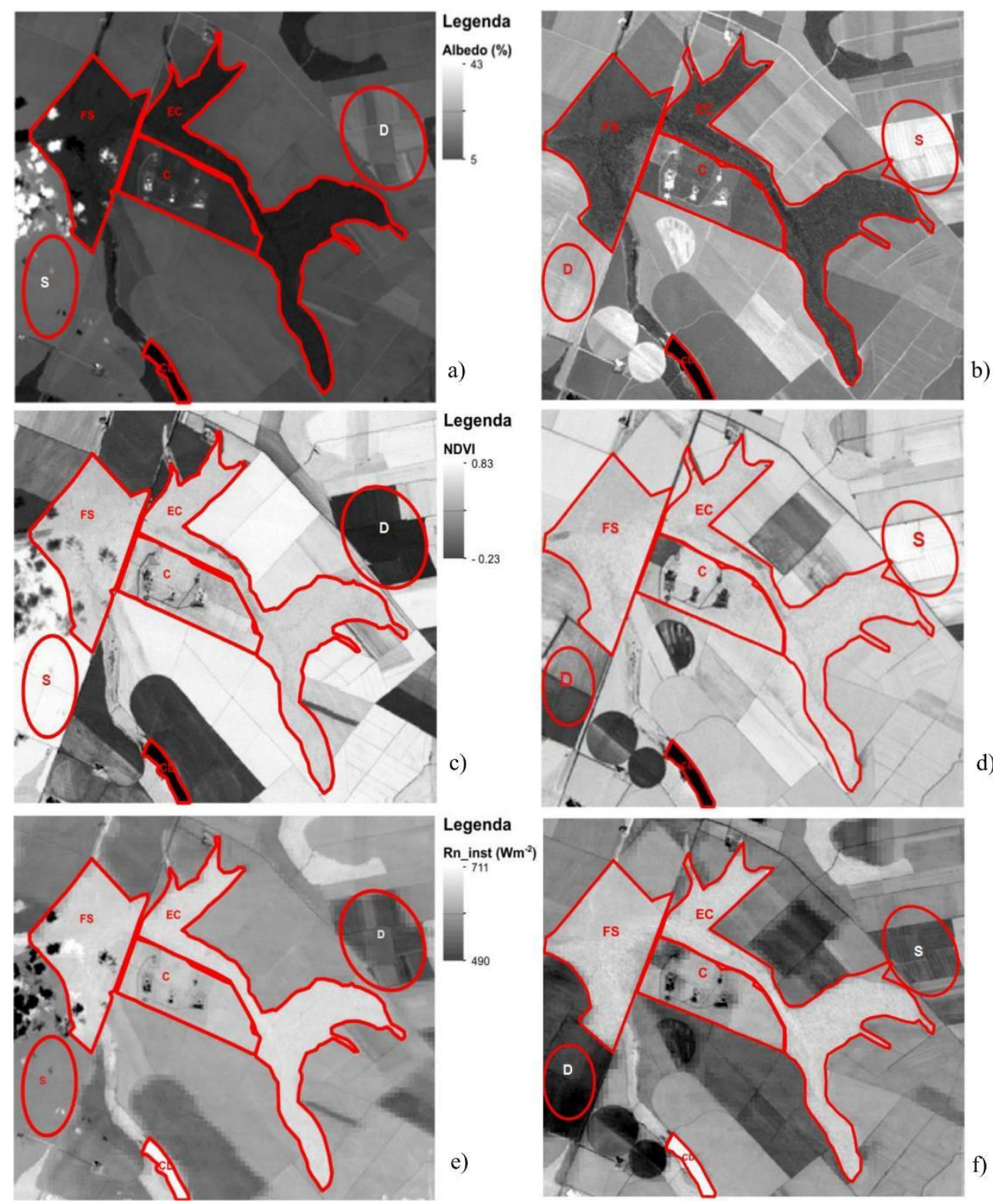

Figura 2. Imagens das componentes Albedo, NDVI e $\mathrm{Rn}_{-i n s t}$ dos dois dias investigados: Dia 20/01/2010: a) Albedo, c) NDVI, e) $\mathrm{Rn}_{\text {_inst.; }}$; Dia 26/01/2010: b) Albedo, d) NDVI, f) Rn_inst. 
Destaca-se que os valores de $\mathrm{Rn}_{\text {inst }}$ mantiveram-se altos frente às outras áreas, podendo inferir maior taxa evapotranspirativa, uma vez que neste período a região estudada não sofre com falta d'água. A substituição em larga escala dessas florestas por culturas poderá ocorrer alterações nos regimes de chuvas, uma vez que, o ritmo temporal do saldo de radiação, albedo, NDVI e outras variáveis são modificados. Costa et al. (2007) simulando a substituição de floresta por cultura de soja na região amazônica encontraram redução de até $16 \%$ nas precipitações para a região. Souza et al. (2007) no estado do Pará, observaram impactos médios anuais de redução de $44 \%$ no calor latente e aumento em $29 \%$ do calor sensível.

Nas demais áreas, percebe-se que ocorreram mudanças nas variáveis, principalmente no albedo da área $\mathrm{C}$ (Tabela 3), que corresponde ao entorno da sede da propriedade com predomínio de solo desnudo, entretanto, altos índices de albedo são característicos para áreas residenciais/urbanas (Santos e Lima, 2013). Usando SEBAL, Oliveira et al. (2012) encontrou valores de albedo maiores que $34 \%$ para região urbana da bacia do rio Tapacurá, Pernambuco.

\section{CONCLUSÃO}

Conclui-se que os valores do $\mathrm{Rn}_{\text {inst }}$, albedo e NDVI apresentaram variações nas áreas com alta sucessão de culturas. A variação nos valores de NDVI em floresta secundária e eucalipto entre janeiro e abril evidencia ganho em biomassa. Os saldos radiativos em áreas de florestas secundárias e eucalipto são maiores que os obtidos para áreas de culturas em período de pleno desenvolvimento, embora esse período, para as culturas, seja temporário no decorrer do ano.

\section{REFERÊNCIAS}

ALLEN, R. G.; TREZZA, R.; TASUMI, M. Surface energy balance algorithms for land: advance training and user's manual. Version 1.0. [S.1.]: Waters Consulting; University of Idaho; WaterWatch, Inc, 2002. p.98.

ALLEN, R. G.; TASUMI, M.; TREZZA, R. Satellite-based energy balance for mapping evapotranspiration with internalized calibration (METRIC) - Model. Journal of Irrigation and Drainage Engineering, v. 133, p. 380-394, 2007. http://dx.doi.org/10.1061/(ASCE)0733-9437(2007)133:4(380)

BASTIAANSSEN, W. G. M. Regionalization of surface flux densities and moisture indicators in composite terrain. 1995. 273f. Ph.D. (Thesis) - Wageningen Agricultural University, Wageningen, Netherlands, 1995.

BASTIAANSSEN, W. G. M. SEBAL - based sensible and latent heat fluxes in the irrigated Gediz Basin, Turkey. Journal of Hydrology, v. 229, p. 87-100, 2000. http://dx.doi.org/10.1016/S0022-1694(99)00202-4

BASTIAANSSEN, W. G. M.; MENENTI, M.; FEDDES, R. A.; HOLTSLAG, A. A. M. A remote sensing surface energy balance algorithm for land (SEBAL) 1. Formulation. Journal of Hydrology, v. 212/213, p. 198-212, 1998. http://dx.doi.org/10.1016/S00221694(98)00253-4

BECKMANN, E.; OLIVEIRA, A. M. Levantamento e análise do potencial agroindustrial dos municípios mato-grossenses de Sinop, Sorriso e Lucas do Rio Verde. Revista de Estudos Sociais, n. 24, v. 2, 2010. 
CHANDER, G.; MARKHARM, B. Revised Landsat 5 - TM radiometric calibration procedures and post calibration dynamic ranges. IEEE Transactions on Geosciences and Remote Sensing, v. 41, p. 2674-2677, 2003. http://dx.doi.org/10. 1109/TGRS.2003.818464

COSTA, M. H.; YANAGY, S. M. N; SOUZA, P. J. O. P.; RIBEIRO, A.; ROCHA, E. J. P. Climate change in Amazonia caused by soybean cropland expansion, as compared to caused by pastureland expansion. Geophysical Research Letters, v. 34, L07706, 2007. http://dx.doi.org/10.1029/2007GL029271

DUBREUIL, V.; DELAHAYE, C. Changements d'occupation du sol et leurs impacts climatiques au Mato Grosso, Brésil. França. Confins, v. 10, 2010.

FERREIRA, J. C. V. Mato Grosso e seus municípios. Cuiabá: Secretaria de Estado de Educação, 2001.

FONTANA, D. C.; BERLATO, M. A.; BERGAMASCHI, H. Balanço de radiação da soja em região subtropical do Brasil. Pesquisa Agropecuária Brasileira, v. 26, n. 3, p. 411418, 1991.

GOMES, H. F.; SILVA, B. B.; CAVALCANTI, E. P.; ROCHA, H. R. Balanço de radiação em diferentes biomas no Estado de São Paulo mediante imagens Landsat 5. São Paulo, UNESP. Geociências, v. 28, n. 2, p. 153-164, 2009.

GUSMÃO, A. C. V. L.; SILVA, B. B.; MONTENEGRO, S. M. G. L.; GALVÍNCIO, J. D. Determinação do saldo radiativo na Ilha do Bananal/TO, com imagens orbitais. Revista Brasileira de Engenharia Agrícola e Ambiental, v. 16, n. 10, p. 1107-1114, 2012. http://dx.doi.org/10.1590/S1415-43662012001000011

HUETE, A.; DIDAN, K.; MIURA, T.; RODRIGUEZ, E. P.; GAO, X.; FERREIRA, L. G. Overview of the radiometric and biophysical performance of the MODIS vegetation indices. Remote Sensing of Environment, v. 83, p. 195-213, 2002. http://dx.doi.org/10.1016/S0034-4257(02)00096-2

HUETE, A. R. A soil adjusted vegetation index (SAVI). Remote Sensing of Environment, v. 25, p. 295-309, 1988. http://dx.doi.org/10.1016/0034-4257(88)90106-X

INSTITUTO BRASILEIRO DE GEOGRAFIA E ESTATÍSTICA (IBGE). Censo demográfico do Brasil. Rio de Janeiro, 2010.

JACOBS, A. F. G.; VAN PUL, W. A. J. Seasonal changes in the Albedo of a Maize Crop during two Seasons. Agricultural and Forest Meteorology, v. 49, p. 351-360, 1990. http://dx.doi.org/10.1016/0168-1923(90)90006-R

KAR, G.; KUMAR, A. Energy balance and crop water stress in winter maize under phenology-based irrigation scheduling. Irrigigation Science, v. 28, p. 211-220, 2010. http://dx.doi.org/10.1007/s00271-009-0192-x

MARKHAM, B. L.; BARKER, J. L. Landsat MSS and TM post-calibration dynamic ranges, exoatmospheric reflectances and at-satellite temperatures. Lanham: Earth Observation Satellite Co., 1986. 
MORSE, A.; ALLEN, R. G.; TASUMI, M.; KRAMBER, W. J.; TREZZA, R.; WRIGHT, J. Application of the SEBAL Methodology for Estimating Evapotranspiration and consumptive use of water through remote sensing. Final Report. [S.1.]: Idaho Department of Water Resources, 2000. 220 p.

OLIVEIRA, G.; MORAES, E. C. Validação do balanço de radiação obtido a partir de dados MODIS/TERRA na Amazônia com medidas de superfície do LBA. Acta Amazonica, v. 43, n. 3, p. 353-364, 2013. http://dx.doi.org/10.1590/S0044-59672013000300011

OLIVEIRA, L. M. M.; MONTENEGRO, S. M. G. L.; ANTONINO, A. C. D.; SILVA, B. B.; MACHADO, C. C. C.; GALVÍNCIO, J. D. Análise quantitativa de parâmetros biofísicos de bacia hidrográfica obtidos por sensoriamento remoto. Pesquisa Agropecuária Brasileira, Brasília, v. 47, n. 9, p. 1209-1217, set. 2012. http://dx.doi.org/10.1590/S0100-204X2012000900005

SANTOS, C. A. C.; LIMA, J. R. A. Análise dos efeitos da expansão urbana de Manaus-AM sobre parâmetros ambientais através de imagens de satélite. Revista Brasileira de Geografia Física, v. 06, n. 01, p. 001-014, 2013.

SEIXAS, G. B.; SOBRINHO, F. J. C.; SANTANA, F. B.; BIUDES, M. S.; NOGUEIRA, J. S. Estimativa do albedo em área de cambarazal no pantanal norte utilizando o algoritimo SEBAL e imagens TM/LANDSAT 5. In: SIMPÓSIO DE GEOTECNOLOGIAS NO PANTANAL, 3., 16-20 de outubro, Cáceres. Anais... São José dos Campos: Embrapa Informática Agropecuária; INPE, 2010. p. 144 -150

SILVA NETO, J. L.; SOUZA, J. L.; SILVA, P. T.; TEODORO, I.; SANTOS, E. A.; AMORIM, E. C. et al. Análise dos componentes de radiação para três condições de plantio de milho. Maceió: Universidade Federal de Alagoas, 2008.

SILVA, B. B.; BRAGA, A. C.; BRAGA, C. C. Balanço de radiação no perímetro irrigado São Gonçalo/PB mediante imagens orbitais. Revista Caatinga, v. 24, p. 145-152, 2011.

SILVA, B. B.; LOPES, G. M.; AZEVEDO, P. V. Determinação do albedo de áreas irrigadas com base em imagens Landsat 5 - TM. Revista Brasileira de Agrometeorologia, v. 13, p. 201-211, 2005a.

SOUZA, P. J. O. P.; RIBEIRO, A.; ROCHA, E. J. P.; LOUREIRO, R. S.; BISPO, C. J. C.; SOUSA, A. M. L. Balanço de energia na soja em áreas de fronteira agrícola na Amazônia. In: CONGRESSO BRASILEIRO DE AGROMETEOROLOGIA, 15., 2007, Aracaju. Anais... [S.1.]: SBAGRO, 2007. 1 CD-ROM.

SOUZA, P. J. O. P.; RIBEIRO, A.; ROCHA, E. J. P.; LOUREIRO, R. S.; BISPO, C. J.; SOUSA, A. M. L. Albedo da cultura da soja em área de avanço da fronteira agrícola na Amazônia. Revista Brasileira de Engenharia Agrícola e Ambiental, v. 14, n. 1, p. 65-73, 2010.

SOUZA, P. J. O. P.; ROCHA, E. J. P.; RIBEIRO, A. Impactos do avanço da soja no balanço de radiação no leste da Amazônia. Acta Amazônica, v. 43, n. 2, p. 169-178, 2013. http://dx.doi.org/10.1590/S0044-59672013000200006

TIAN, F.; QIU, G.Y.; YANG, Y.H.; XIONG, Y.J.; WANG, P. Studies on the Relationships Between Land Surface Temperature and Environmental Factors in an Inland River Catchment Based on Geographically Weighted Regression and MODIS Data. IEEE Journal of selected topics in applied earth observations and remote sensing, v. 5, n. 3, p. , Jun. 2012. http://dx.doi.org/10.1109/JSTARS.2012.2190978 
WIKIMEDIA COMMONS. Map locator of Mato Grosso's Lucas do Rio Verde city. 20 Jan. 2010. Disponível em: http://wikimedia.org/MatoGrosso_Municip_Lucasdo RioVerde.svg/200px-.svg.png>. Acesso em: 30 dez. 2013 